\title{
Splenomegaly and Its Associations with Genetic Polymorphisms and Treatment Outcome in Colorectal Cancer Patients Treated with Adjuvant FOLFOX
}

\author{
Mi-Jung Kim, $\mathrm{MD}^{1,23}$ \\ Sae-Won Han, MD, PhD',2 \\ Dae-Won Lee, MD ${ }^{1}$ \\ Yongjun Cha, MD \\ Kyung-Hun Lee, MD ${ }^{1,2}$ \\ Tae-Yong Kim, MD',2 \\ Do-Youn Oh, MD, PhD',2 \\ Se Hyung Kim, MD, PhD ${ }^{4}$ \\ Seock-Ah Im, MD, PhD',2 \\ Yung-Jue Bang, MD, PhD',2 \\ Tae-You Kim, MD, PhD ${ }^{1,2}$
}

\begin{abstract}
Purpose
Splenomegaly is a clinical surrogate of oxaliplatin-induced sinusoidal obstruction syndrome (SOS). We investigated development of splenomegaly and its association with treatment outcome and genetic polymorphisms following adjuvant 5-fluorouracil, leucovorin, and oxaliplatin (FOLFOX) in colorectal cancer (CRC) patients.
\end{abstract}

\section{Materials and Methods}

Splenomegaly was determined by spleen volumetry using computed tomography images obtained before initiation of chemotherapy and after completion of adjuvant FOLFOX in CRC patients. Ten genetic polymorphisms in 4 SOS-related genes (VEGFA, MMP9, NOS3, and GSTP1) were analyzed using DNA from peripheral blood mononuclear cells.

\section{Results}

Of 124 patients included, increase in spleen size was observed in 109 (87.9\%). Median change was $31 \%$ (range, $-42 \%$ to $168 \%$ ). Patients with splenomegaly had more severe thrombocytopenia compared to patients without splenomegaly during the chemotherapy period $(p<0.0001)$. The cumulative dose of oxaliplatin and the lowest platelet count during the chemotherapy period were clinical factors associated with splenomegaly. However, no significant associations were found between genetic polymorphisms and development of splenomegaly. Disease-free survival was similar regardless of the development of splenomegaly.

\section{Conclusion}

Splenomegaly was frequently observed in patients receiving adjuvant FOLFOX and resulted in more severe thrombocytopenia but did not influence treatment outcome. Examined genetic polymorphisms did not predict development of splenomegaly.
Correspondence: Sae-Won Han, MD, PhD Department of Internal Medicine, Seoul National University Hospital, 101 Daehak-ro, Jongno-gu, Seoul 03080, Korea Tel: 82-2-2072-0242

Fax: 82-2-762-9662

E-mail: saewon1@snu.ac.kr

Received August 6, 2015

Accepted December 19, 2015

Published Online January 14, 2016

\section{Key words}

Colorectal neoplasms, Oxaliplatin,

Sinusoidal obstruction syndrome, Splenomegaly, Genetic polymorphism 


\section{Introduction}

Colorectal cancer (CRC) is a common malignancy and a leading cause of cancer death worldwide [1], and the incidence of CRC is increasing rapidly in Eastern countries including Korea as a result of recent changes in diet and lifestyle [2]. The primary treatment of CRC is a complete resection in stage I-III patients followed by adjuvant chemotherapy in stage III and high-risk stage II patients. In the considerable number of patients with distant metastases (i.e., stage IV disease) at the time of diagnosis, chemotherapy is the mainstay of treatment and selected patients with resectable metastases undergo surgery. Complete resection of colorectal liver metastases can provide a chance for longterm survival in approximately $20 \%$ of these patients [3].

Oxaliplatin plus fluoropyrimidine combination chemotherapy is widely used in both the adjuvant and palliative setting to improve survival of CRC patients [4,5]. With increasing incorporation of liver metastasectomy in the treatment strategy, many patients treated with oxaliplatin-containing chemotherapy undergo hepatic resection. Sinusoidal obstruction syndrome (SOS) in the liver is a long-term toxicity of oxaliplatin reported in patients who underwent hepatic resection after oxaliplatin-based chemotherapy [6-10].

SOS, previously known as hepatic veno-occlusive disease, was most commonly reported to occur after bone marrow transplantation for hematologic malignancies [11]. SOS has been reported in 19\%-79\% of CRC patients who underwent hepatic resection after oxaliplatin-based chemotherapy $[6,7,12]$. Toxic effect of oxaliplatin on sinusoidal endothelial cells (SEC) causes disruption of the sinusoidal wall, subsequently causing congestive obstruction with impairment of sinusoidal blood flow [13]. As a result, diffuse sinusoidal injury leads to portal hypertension, hepatomegaly, and hyperbilirubinemia with severe complications such as ascites and variceal bleeding in rare cases [14]. In addition, development of SOS may be associated with increased morbidity and mortality following hepatic resection in patients treated with preoperative oxaliplatin-based regimens [7].

Prediction of SOS development is important in selection of proper candidates for hepatic resection and preoperative management of patients at risk of SOS. Increase in spleen size, elevated aspartate aminotransferase to platelet ratio index, and hyaluronic acid levels have been reported as reliable indicators of SOS $[9,15,16]$. However, there is no reliable biomarker for prediction of SOS before oxaliplatin treatment that could help in the decision of oxaliplatin use. In addition, association of the susceptibility to SOS with antitumor efficacy in the adjuvant setting has not been studied.

Many recent efforts have been made to clarify the pathogenesis of SOS at the molecular level and to use this as a molecular marker of SOS. A rat model based on monocrotaline gavage by Deleve et al. [17] suggested that SEC injury is a major initiating event of SOS. This model also suggested that additional mechanisms such as an increase in expression of matrix metalloproteinase-9 (MMP-9) (and to a lesser extent MMP-2), reduced synthesis in nitric oxide, and oxidative stress contribute to SEC injury. Vascular endothelial growth factor (VEGF) is known to regulate MMP-9 activation by inducing its expression, and the degree of the increase in VEGF serum level parallels the clinical severity of SOS [18]. Therefore, VEGF blockade may attenuate sinusoidal injury by down-regulating MMP-9 production [19]. In the liver, nitric oxide is produced by the nitric oxide synthase 3 (NOS3) expressed in the SEC. Decreased activity of glutathione S-transferase (GST) leads to a decrease of adduct formation between glutathione and platinum, consequently attenuating a defense mechanism against oxaliplatin. In this study, we have chosen several genes related to the pathogenesis of SOS (VEGFA, MMP9, NOS3, and GSTP1), as shown in the previous model, and studied the association between their genetic polymorphisms and SOS following oxaliplatin treatment using spleen size as a surrogate of SOS. CRC patients receiving adjuvant 5-fluorouracil, leucovorin, and oxaliplatin (FOLFOX) were selected in order to eliminate the potential bias caused by the extent of liver metastasis. We also analyzed the association of SOS with disease-free survival (DFS) following adjuvant FOLFOX.

\section{Materials and Methods}

\section{Study population and treatment}

This retrospective analysis included stage III or high-risk stage II CRC patients who received adjuvant FOLFOX chemotherapy after complete resection of CRC from September 2005 to December 2009 at Seoul National University Hospital (SNUH) and participated in pharmacogenomics study of chemotherapy among cancer patients. Other eligibility criteria were age over 18 years, adenocarcinoma histology, complete resection of primary tumor with negative margin, adequate organ function, completion of at least six cycles of the planned 12 cycles of chemotherapy, and computed tomography (CT) images obtained before and after chemotherapy adequate for measurement of spleen size. For adequate measurement, contrast-enhanced CT images were obtained with at least $5 \mathrm{~mm}$ slice thickness or less. Patients with underlying severe liver disease such as active viral hepatitis, severe steatohepatitis, or liver cirrhosis before chemotherapy were excluded. 
Patients received a maximum of 12 cycles of FOLFOX- 4 or modified FOLFOX-6. Each cycle of FOLFOX-4 consisted of oxaliplatin $\left(85 \mathrm{mg} / \mathrm{m}^{2}\right)$ on day 1 and leucovorin $\left(200 \mathrm{mg} / \mathrm{m}^{2}\right)$ and a bolus of 5-fluorouracil (5-FU; $400 \mathrm{mg} / \mathrm{m}^{2}$ ) followed by a 22-hour infusion of 5 -FU $\left(600 \mathrm{mg} / \mathrm{m}^{2}\right)$ on days 1 and 2, which was repeated every 2 weeks. Modified FOLFOX-6 consisted of oxaliplatin $\left(85 \mathrm{mg} / \mathrm{m}^{2}\right)$, leucovorin $\left(400 \mathrm{mg} / \mathrm{m}^{2}\right)$, and a bolus of 5 -FU $\left(400 \mathrm{mg} / \mathrm{m}^{2}\right)$ followed by a 46 -hour infusion of 5 -FU $\left(2,400 \mathrm{mg} / \mathrm{m}^{2}\right)$ repeated every 2 weeks. All patients gave informed consent to the pharmacogenomics study prior to peripheral blood collection. The study protocol was reviewed and approved by the institutional review board of SNUH and was conducted in accordance with the Declaration of Helsinki.

\section{Genotyping}

Genomic DNA was extracted from peripheral blood mononuclear cells using a QIAamp DNA kit (Qiagen, Valencia, CA), and each polymorphism was determined using a pyrosequencing method (PyroMark Q96 ID, Qiagen). Primer sequence and biological effect of each polymorphism are shown in Supplementary Table 1. Ten polymorphisms in 4 potentially SOS-related genes were analyzed: $-2578 \mathrm{C} / \mathrm{A}$, $-1154 \mathrm{G} / \mathrm{A},-634 \mathrm{G} / \mathrm{C}$, and $936 \mathrm{C} / \mathrm{T}$ in VEGFA; $-1562 \mathrm{C} / \mathrm{T}$, 836A/G, and 2003G/A in MMP9; -786T/C and 894G/T in NOS3, and Ile105Val in GSTP1.

\section{Spleen size measurement}

Spleen size was measured by loading the $\mathrm{CT}$ images into a 3D Workstation (Rapidia ver. 2.8, Infinitt Healthcare, Seoul, Korea) and using volume viewer software. The outline of the spleen on each axial image of CT scans was traced using an electronic free-curve in the software, cross-sectional areas were calculated, and then the sum of the areas was multiplied by slice thickness for calculation of spleen volume. Spleen sizes measured from CT images obtained before chemotherapy and after completion of chemotherapy were compared.

\section{Statistical analysis}

Association between change in spleen size and each genetic polymorphism was analyzed using Student's t test. The relationship between splenomegaly and each polymorphism was also analyzed using Pearson's chi-square or Fisher's exact test. Splenomegaly was functionally defined as a $\geq 50 \%$ increase in spleen size after oxaliplatin-based chemotherapy.

Multivariate logistic regression analysis was performed to evaluate the effect of other clinicopathologic factors on
Table 1. Patient characteristics

$\begin{array}{lc}\text { Characteristic } & \text { No. }(\%)(\mathbf{n}=124) \\ \text { Age } & \\ \quad \text { Median (range, yr) } & 60(30-76) \\ \text { Sex } & \\ \quad \text { Male } & 73(58.9) \\ \quad \text { Female } & 51(41.1) \\ \text { Primary site } & \\ \quad \text { Proximal } & 36(29.0) \\ \quad \text { Distal } & 88(71.0) \\ \text { Stage } & \\ \quad \text { II } & 16(12.9) \\ \quad \text { III } & 108(87.1) \\ \left.\text { Underlying chronic liver disease }{ }^{a}\right) & 21(16.9) \\ \text { Cycle of chemotherapy } & 12(6-12) \\ \quad \text { Median }(\text { range }) & \\ \text { Cumulative dose of oxaliplatin } & 54(43.5) \\ \quad \text { Full }\left(1,020 \mathrm{mg} / \mathrm{m}^{2}\right) & 70(56.5) \\ \quad \text { Reduced }\left(<1,020 \mathrm{mg} / \mathrm{m}^{2}\right) & \end{array}$

${ }^{a}$ Chronic liver disease included chronic inactive viral hepatitis such as hepatitis B and C, nonalcoholic steatohepatitis and fatty liver diseases.

splenomegaly. In this analysis, the backward stepwise regression model including only variables with a p-value $<0.10$ in univariate analysis was used. Survival functions for DFS were estimated using the Kaplan-Meier method, and differences between groups were tested using a log-rank test. Two-sided $p$-values of $<0.05$ were considered significant. Analyses were performed using SPSS ver. 17.0 (SPSS Inc., Chicago, IL).

\section{Results}

\section{Patient characteristics and changes in spleen size}

Of a total of 124 patients included in this study, 73 patients $(58.9 \%)$ were male (Table 1$)$. The median age was 60 years (range, 30 to 76 years). Patients received median 12 cycles (range, 6 to 12 cycles) of adjuvant FOLFOX; 108 patients $(87.1 \%)$ completed all 12 cycles of adjuvant FOLFOX. The median cumulative dose of oxaliplatin was $935 \mathrm{mg} / \mathrm{m}^{2}$ (range, 459 to $1,020 \mathrm{mg} / \mathrm{m}^{2}$ ).

Increase in spleen size was observed in 109 patients (87.9\%) after completion of chemotherapy compared with baseline size before chemotherapy. The median change in 
Table 2. Associations between genetic polymorphisms and change in spleen size

\begin{tabular}{|c|c|c|c|c|c|}
\hline \multirow{2}{*}{ Polymorphism } & \multirow{2}{*}{ No. $(\%)$} & \multicolumn{2}{|c|}{ Change in spleen size } & \multicolumn{2}{|c|}{ Splenomegalya) } \\
\hline & & Mean $\%$ & p-value $e^{\text {b) }}$ & No. $(\%)$ & p-value \\
\hline \multicolumn{6}{|l|}{ VEGFA C2578A } \\
\hline $\mathrm{CC}$ & $69(55.6)$ & $38.6 \pm 39.3$ & 0.86 & $28(40.6)$ & 0.49 \\
\hline CA or AA & $55(44.4)$ & $39.8 \pm 37.5$ & & $19(34.5)$ & \\
\hline \multicolumn{6}{|l|}{ VEGFA C634G } \\
\hline $\mathrm{CC}$ & $22(17.7)$ & $45.8 \pm 32.3$ & 0.37 & $9(40.9)$ & 0.75 \\
\hline CG or GG & $102(82.3)$ & $37.7 \pm 39.5$ & & $38(37.3)$ & \\
\hline \multicolumn{6}{|l|}{ VEGFA C936T } \\
\hline $\mathrm{CC}$ & $75(60.5)$ & $38.6 \pm 37.9$ & 0.85 & $27(36.0)$ & 0.59 \\
\hline CT or TT & $49(39.5)$ & $39.9 \pm 39.5$ & & $20(40.8)$ & \\
\hline \multicolumn{6}{|l|}{ VEGFA G1154A } \\
\hline GG & $85(68.5)$ & $37.2 \pm 39.2$ & 0.42 & $31(36.5)$ & 0.63 \\
\hline GA or AA & $39(31.5)$ & $43.2 \pm 36.8$ & & $16(41.0)$ & \\
\hline \multicolumn{6}{|l|}{ MMP9 C1562T } \\
\hline $\mathrm{CC}$ & $87(70.2)$ & $37.8 \pm 36.8$ & 0.56 & $30(34.5)$ & 0.23 \\
\hline CT or TT & $37(29.8)$ & $42.2 \pm 42.2$ & & $17(45.9)$ & \\
\hline \multicolumn{6}{|l|}{ MMP9 A836G } \\
\hline AA & $19(15.3)$ & $42.8 \pm 36.6$ & 0.65 & $7(36.8)$ & 0.92 \\
\hline AG or GG & $105(84.7)$ & $38.4 \pm 38.8$ & & $40(38.1)$ & \\
\hline \multicolumn{6}{|l|}{ MMP9 G2003A } \\
\hline GG & $87(70.2)$ & $37.8 \pm 36.8$ & 0.56 & $30(34.5)$ & 0.23 \\
\hline GA or AA & $37(29.8)$ & $42.2 \pm 42.2$ & & $17(45.9)$ & \\
\hline \multicolumn{6}{|l|}{ NOS3 G894T } \\
\hline GG & $104(83.9)$ & $38.6 \pm 39.0$ & 0.74 & $39(37.5)$ & 0.83 \\
\hline GT or TT & $20(16.1)$ & $41.8 \pm 35.5$ & & $8(40.0)$ & \\
\hline \multicolumn{6}{|l|}{ NOS3 T786C } \\
\hline TT & $101(81.5)$ & $37.8 \pm 37.8$ & 0.43 & $36(35.6)$ & 0.28 \\
\hline $\mathrm{TC}$ & $23(18.5)$ & $44.8 \pm 41.3$ & & $11(47.8)$ & \\
\hline \multicolumn{6}{|l|}{ GSTP1 Ile105Val } \\
\hline Ile/Ile & $71(57.3)$ & $37.8 \pm 40.8$ & 0.65 & $25(35.2)$ & 0.48 \\
\hline Ile/Val or Val/Val & $53(42.7)$ & $40.9 \pm 35.2$ & & $22(41.5)$ & \\
\hline
\end{tabular}

VEGFA, vascular endothelial growth factor A; MMP, matrix metalloproteinase; NOS, nitric oxide synthase; GST, glutathione S-transferase. ${ }^{\text {a) }}$ Splenomegaly was defined as a $\geq 50 \%$ increase in spleen size after oxaliplatin-based chemotherapy, bistudent's $\mathrm{t}$ test was used, 'Pearson's chi square or Fisher's exact tests were used.

spleen size was $31 \%$ (range, $-42 \%$ to $168 \%$ ). Splenomegaly ( $\geq 50 \%$ increase) was observed in 47 patients $(37.9 \%)$. The mean increase in spleen size was higher in patients receiving a full dose of oxaliplatin during 12 cycles $(n=54)$ compared with patients receiving a reduced dose of oxaliplatin $(\mathrm{n}=70)$ (50.7 $\pm 40.4 \%$ vs. $30.2 \pm 34.4 \%, \mathrm{p}=0.003)$.

\section{Factors associated with splenomegaly}

Ten genetic polymorphisms within four genes were analyzed (VEGFA, MMP9, NOS3, and GSTP1). When the relationship between these genetic polymorphisms and change in spleen size or splenomegaly was analyzed in univariate analysis, no genetic polymorphism was associated with change in spleen size or splenomegaly ( $p$-value, not significant) (Table 2).

In univariate analysis for development of splenomegaly, only the cumulative dose of oxaliplatin (full vs. reduced dose) was significantly associated with development of splenomegaly. No significant associations were found between splenomegaly and other clinical factors (age [ $>65$ years vs. $\leq 65$ years], lowest platelet count during chemotherapy $\left[<75,000 / \mathrm{mm}^{3}\right.$ vs. $\left.\geq 75,000 / \mathrm{mm}^{3}\right]$ and the presence of chronic liver disease). In multivariate logistic regression analysis performed using variables with $p$-values $<0.10$ in univariate analysis, the cumulative dose of oxaliplatin and 
Table 3. Univariate and multivariate analyses of development of splenomegaly

\begin{tabular}{|c|c|c|c|c|}
\hline \multirow{2}{*}{ Characteristic } & \multicolumn{2}{|c|}{ Univariate analysis } & \multicolumn{2}{|c|}{ Multivariate analysis } \\
\hline & No. $(\%)$ & p-value & OR $(95 \% \mathrm{CI})$ & p-value \\
\hline \multicolumn{5}{|l|}{ Age } \\
\hline$\leq 65 \mathrm{yr}$ & $38(41.8)$ & 0.142 & - & - \\
\hline$>65 \mathrm{yr}$ & $9(27.3)$ & & - & \\
\hline \multicolumn{5}{|c|}{ Oxaliplatin cumulative dose } \\
\hline Reduced dose & $19(27.1)$ & 0.005 & 1 (reference) & 0.003 \\
\hline Full dose & $28(51.9)$ & & $3.29(1.50-7.18)$ & \\
\hline \multicolumn{5}{|c|}{ The lowest PLT count during chemotherapy } \\
\hline$\geq 75,000 / \mathrm{mm}^{3}$ & $30(33.3)$ & 0.088 & 1 (reference) & 0.040 \\
\hline$<75,000 / \mathrm{mm}^{3}$ & $17(50.0)$ & & $2.45(1.04-5.76)$ & \\
\hline \multicolumn{5}{|c|}{ Presence of chronic liver disease ${ }^{a)}$} \\
\hline No & $42(40.8)$ & 0.144 & - & - \\
\hline Yes & $5(23.8)$ & & - & \\
\hline
\end{tabular}

OR, odds ratio; $\mathrm{CI}$, confidence interval; PLT, platelet. a) Chronic liver disease included chronic inactive viral hepatitis such as hepatitis $\mathrm{B}$ and $\mathrm{C}$, nonalcoholic steatohepatitis and fatty liver diseases.

the lowest platelet count during the chemotherapy period were associated with splenomegaly (Table 3).

\section{Splenomegaly and thrombocytopenia}

During adjuvant FOLFOX chemotherapy and the followup period after completion of chemotherapy, patients with splenomegaly had lower values of mean platelet count compared to patients without splenomegaly. This difference in mean platelet count was the most prominent from 3 months to 6 months after initiation of FOLFOX ( $p<0.05$, by Student's $t$ test), and the difference was gradually reduced after completion of chemotherapy (Fig. 1). In addition, patients with splenomegaly had more severe thrombocytopenia compared to those without splenomegaly during the chemotherapy period (mean lowest platelet count, $85,000 \pm 28,000 / \mathrm{mm}^{3} \mathrm{vs}$. $\left.115,000 \pm 42,000 / \mathrm{mm}^{3} ; \mathrm{p}<0.001\right)$.

\section{Splenomegaly and DFS}

We also analyzed the association of splenomegaly, a surrogate marker of SOS, with treatment outcome of adjuvant FOLFOX chemotherapy. DFS was similar according to development of splenomegaly. The 3-year DFS rate was $89.1 \%$ (95\% confidence interval [CI], $79.9 \%$ to $98.3 \%$ ) in patients who developed splenomegaly and $85.7 \%$ (95\% CI, $77.7 \%$ to 93.7\%) in patients without splenomegaly ( $\mathrm{p}=0.42$ by log-rank test) (Fig. 2).

\section{Discussion}

In the current study, splenomegaly, a surrogate of SOS, was frequently observed after adjuvant FOLFOX chemotherapy in CRC patients. An increase in spleen size compared with baseline size before starting oxaliplatin-based chemotherapy was observed in 109 patients $(87.9 \%)$, with a median increase in spleen size of $31 \%$. Although the direct comparison was difficult due to the difference in the cumulative dose of oxaliplatin and treatment duration every study, this change in spleen size is comparable to that reported in another study [9].

The cumulative dose of oxaliplatin and the lowest platelet count during chemotherapy were clinical factors associated with splenomegaly. Patients with splenomegaly showed more severe thrombocytopenia than patients without splenomegaly during or after oxaliplatin-based chemotherapy. This is in line with a previous study reporting on the relationship between splenomegaly and thrombocytopenia, which suggested splenic sequestration induced by portal hypertension as a possible mechanism of thrombocytopenia in patients with SOS [9]. Thrombocytopenia related to SOS is common, but usually not severe [20]. In this study, thrombocytopenia less than $50,000 \mathrm{~mm}^{3}$ was only observed in five cases and there were no significant bleeding events. Thrombocytopenia due to oxaliplatin-induced SOS can be prolonged until 2-3 years after completion of oxaliplatin treatment [21]. This slow recovery in platelet count was also observed in our study (Fig. 1). Besides these clinical factors, oxaliplatin-induced SOS is infrequently presented with 


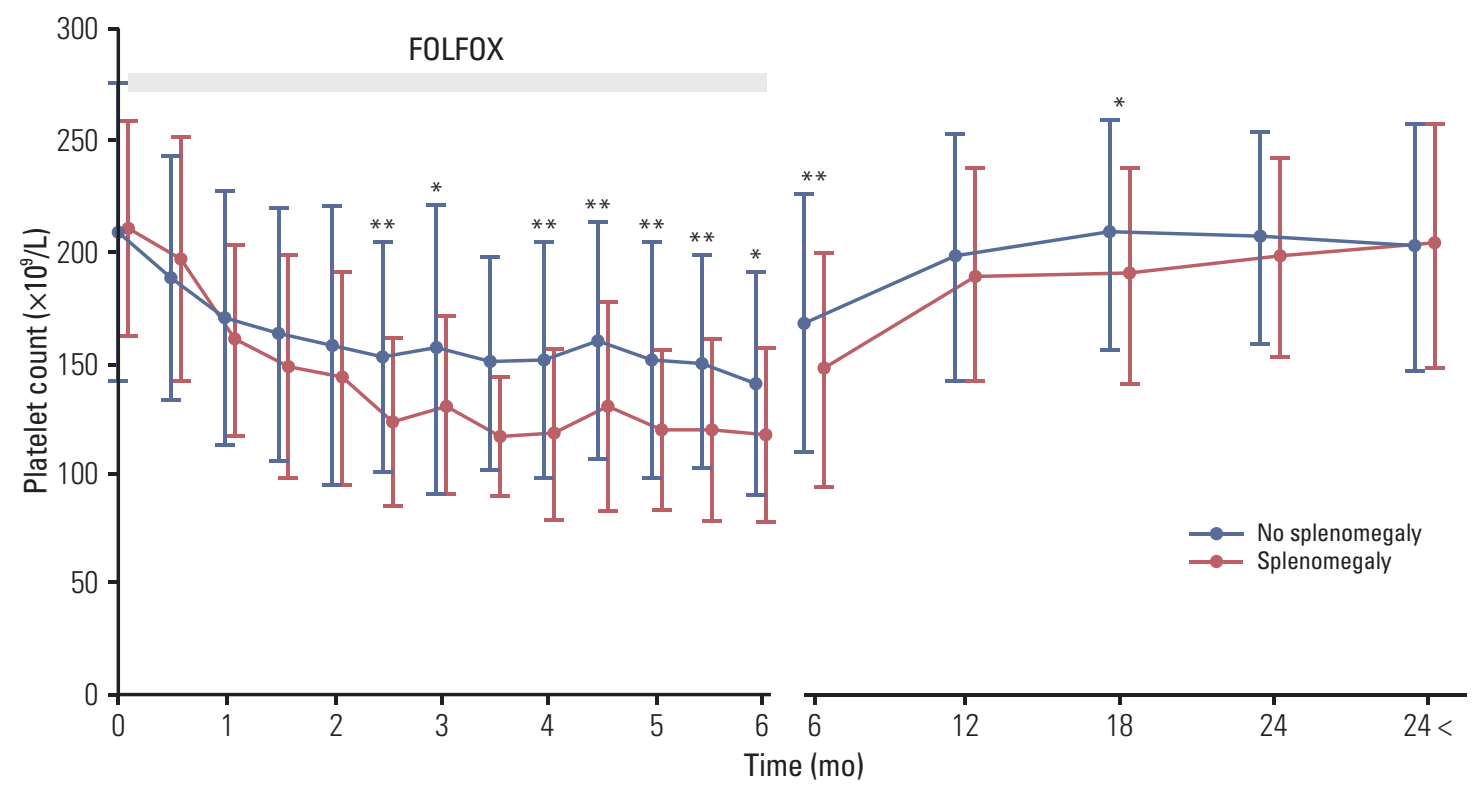

Fig. 1. Changes in platelet counts during or after chemotherapy in patients with or without splenomegaly. FOLFOX, 5-fluorouracil, leucovorin, and oxaliplatin. ${ }^{*} \mathrm{p}<0.05,{ }^{* *} \mathrm{p}<0.01$.

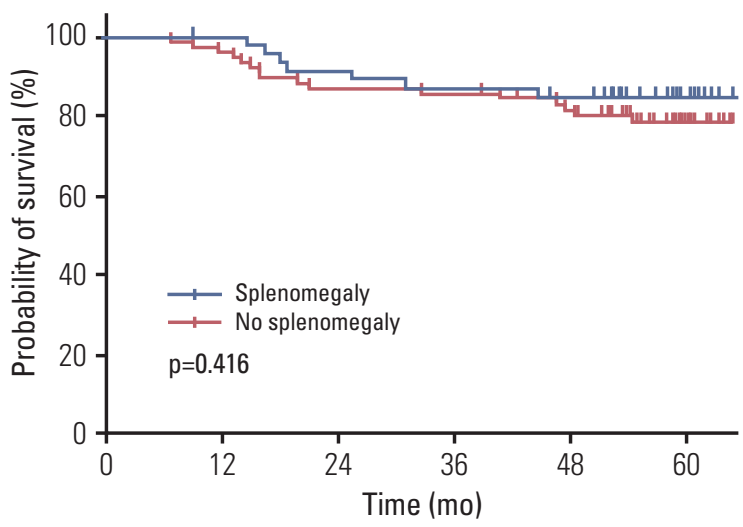

Fig. 2. Kaplan-Meier survival curve of disease-free survival according to the development of splenomegaly.

ascites, jaundice, and hepatomegaly suggestive of portal hypertension [14]. In our study, none of the patients showed clinically apparent symptoms and signs of portal hypertension except splenomegaly.

A number of studies have been conducted for discovery of molecular predictive biomarkers of SOS based on its pathogenesis $[10,22,23]$. Rubbia-Brandt et al. [22], who examined gene expression profiles in livers with oxaliplatin-induced SOS and matched normal controls, found 913 differentially expressed genes. Results of pathway analysis showed signif- icant upregulation of expression in six pathways: acute phase response, coagulation system, hepatic fibrosis / hepatic stellate cell activation, and oxidative stress. In addition, angiogenic and hypoxic factors including VEGFC and hypoxiainducible factor 1-alpha (HIF1A) were upregulated [22]. A similar study conducted by a French group also confirmed the upregulation of genes involved in angiogenesis and coagulation in oxaliplatin-induced sinusoidal injuries [23]. Glutathione forms an adduct with platinum by GST, which leads to detoxification of oxaliplatin. In another study evaluating the role of GST polymorphism as a risk factor for SOS, GSTM1-null genotype was significantly related to the presence of moderate-severe SOS [10].

We have analyzed the association between splenomegaly and genetic polymorphisms in four SOS-related genes. We hypothesized that these genes might be relevant to the pathogenesis of SOS, as suggested in an animal model [13]. In this model, upregulation of MMP-9 and subsequent decrease of nitric oxide contributed to SEC injury, as well as glutathione depletion followed by production of reactive oxygen species. However, we found no association between splenomegaly and the genetic polymorphisms. Further studies are needed to determine the role of other genes or polymorphisms as a risk factor of SOS.

Some studies have suggested a negative impact of oxaliplatin-related SOS on long term outcomes with early recurrence and decreased overall survival in patients with colorectal liver metastases [24]. In addition, more severe 
grade of SOS was correlated with lower histopathological tumor regression [25]. In our study, splenomegaly, used as a biomarker of SOS, showed no association with survival outcome. A possible explanation is that, unlike previous studies, our study was conducted in patients in the adjuvant setting without distant metastasis. In addition, the frequency of hepatic recurrence was not significantly different according to the development of splenomegaly $(2.1 \%$ [1/47] in patients with splenomegaly vs. $5.2 \%$ [4/77] in patients without splenomegaly, $\mathrm{p}=0.65$ ).

The current study has some limitations including the retrospective nature of the study and no histopathological confirmation of SOS. However, the homogeneity of the study population including only patients in the adjuvant setting is the strength of the study.

\section{Conclusion}

In summary, we show that splenomegaly occurred in $87.9 \%$ of CRC patients receiving adjuvant FOLFOX treatment and it was also associated with more severe thrombocytopenia. Importantly, the development of splenomegaly did not affect DFS. As we found no association between the genetic polymorphisms analyzed herein and development of splenomegaly, future studies investigating other biomarker candidates are warranted for prediction of SOS.

\section{Electronic Supplementary Material}

Supplementary materials are available at Cancer Research and Treatment website (http://www.e-crt.otg).

\section{Conflicts of Interest}

Conflict of interest relevant to this article was not reported.

\section{Acknowledgments}

This research was supported by grants from the Korea Health Technology R\&D Project (HI14C1277 and HI13C2163) through the Korea Health Industry Development Institute (KHIDI) funded by the Ministry of Health \& Welfare and the Converging Research Center Program (2014M3C1A8048802) funded by the Ministry of Science, ICT and Future Planning, Republic of Korea.

\section{References}

1. Siegel R, Naishadham D, Jemal A. Cancer statistics, 2012. CA Cancer J Clin. 2012;62:10-29.

2. Jung KW, Won YJ, Kong HJ, Oh CM, Lee DH, Lee JS. Cancer statistics in Korea: incidence, mortality, survival, and prevalence in 2011. Cancer Res Treat. 2014;46:109-23.

3. Choti MA, Sitzmann JV, Tiburi MF, Sumetchotimetha W, Rangsin R, Schulick RD, et al. Trends in long-term survival following liver resection for hepatic colorectal metastases. Ann Surg. 2002;235:759-66.

4. de Gramont A, Figer A, Seymour M, Homerin M, Hmissi A, Cassidy J, et al. Leucovorin and fluorouracil with or without oxaliplatin as first-line treatment in advanced colorectal cancer. J Clin Oncol. 2000;18:2938-47.

5. Andre T, Boni C, Mounedji-Boudiaf L, Navarro M, Tabernero J, Hickish T, et al. Oxaliplatin, fluorouracil, and leucovorin as adjuvant treatment for colon cancer. N Engl J Med. 2004;350: 2343-51.

6. Rubbia-Brandt L, Audard V, Sartoretti P, Roth AD, Brezault C, Le Charpentier M, et al. Severe hepatic sinusoidal obstruc- tion associated with oxaliplatin-based chemotherapy in patients with metastatic colorectal cancer. Ann Oncol. 2004;15: 460-6.

7. Nakano H, Oussoultzoglou E, Rosso E, Casnedi S, ChenardNeu MP, Dufour P, et al. Sinusoidal injury increases morbidity after major hepatectomy in patients with colorectal liver metastases receiving preoperative chemotherapy. Ann Surg. 2008;247:118-24.

8. Angitapalli R, Litwin AM, Kumar PR, Nasser E, Lombardo J, Mashtare T, et al. Adjuvant FOLFOX chemotherapy and splenomegaly in patients with stages II-III colorectal cancer. Oncology. 2009;76:363-8.

9. Overman MJ, Maru DM, Charnsangavej C, Loyer EM, Wang $\mathrm{H}$, Pathak $\mathrm{P}$, et al. Oxaliplatin-mediated increase in spleen size as a biomarker for the development of hepatic sinusoidal injury. J Clin Oncol. 2010;28:2549-55.

10. Vreuls CP, Olde Damink SW, Koek GH, Winstanley A, Wisse E, Cloots RH, et al. Glutathione S-transferase M1-null genotype as risk factor for SOS in oxaliplatin-treated patients with 
metastatic colorectal cancer. Br J Cancer. 2013;108:676-80.

11. Levitsky J, Sorrell MF. Hepatic complications of hematopoietic cell transplantation. Curr Gastroenterol Rep. 2007;9:60-5.

12. Vauthey JN, Pawlik TM, Ribero D, Wu TT, Zorzi D, Hoff PM, et al. Chemotherapy regimen predicts steatohepatitis and an increase in 90-day mortality after surgery for hepatic colorectal metastases. J Clin Oncol. 2006;24:2065-72.

13. Rubbia-Brandt L. Sinusoidal obstruction syndrome. Clin Liver Dis. 2010;14:651-68.

14. Rubbia-Brandt L, Lauwers GY, Wang H, Majno PE, Tanabe K, Zhu AX, et al. Sinusoidal obstruction syndrome and nodular regenerative hyperplasia are frequent oxaliplatin-associated liver lesions and partially prevented by bevacizumab in patients with hepatic colorectal metastasis. Histopathology. 2010;56:430-9.

15. Soubrane O, Brouquet A, Zalinski S, Terris B, Brezault C, Mallet $\mathrm{V}$, et al. Predicting high grade lesions of sinusoidal obstruction syndrome related to oxaliplatin-based chemotherapy for colorectal liver metastases: correlation with post-hepatectomy outcome. Ann Surg. 2010;251:454-60.

16. van den Broek MA, Vreuls CP, Winstanley A, Jansen RL, van Bijnen AA, Dello SA, et al. Hyaluronic acid as a marker of hepatic sinusoidal obstruction syndrome secondary to oxaliplatin-based chemotherapy in patients with colorectal liver metastases. Ann Surg Oncol. 2013;20:1462-9.

17. DeLeve LD, McCuskey RS, Wang X, Hu L, McCuskey MK, Epstein RB, et al. Characterization of a reproducible rat model of hepatic veno-occlusive disease. Hepatology. 1999;29: 1779-91.

18. Iguchi A, Kobayashi R, Yoshida M, Kobayashi K, Matsuo K, Kitajima I, et al. Vascular endothelial growth factor (VEGF) is one of the cytokines causative and predictive of hepatic veno- occlusive disease (VOD) in stem cell transplantation. Bone Marrow Transplant. 2001;27:1173-80.

19. Ribero D, Wang H, Donadon M, Zorzi D, Thomas MB, Eng C, et al. Bevacizumab improves pathologic response and protects against hepatic injury in patients treated with oxaliplatinbased chemotherapy for colorectal liver metastases. Cancer. 2007;110:2761-7.

20. Jung EJ, Ryu CG, Kim G, Kim SR, Park HS, Kim YJ, et al. Splenomegaly during oxaliplatin-based chemotherapy for colorectal carcinoma. Anticancer Res. 2012;32:3357-62.

21. Jardim DL, Rodrigues CA, Novis YA, Rocha VG, Hoff PM. Oxaliplatin-related thrombocytopenia. Ann Oncol. 2012;23: 1937-42.

22. Rubbia-Brandt L, Tauzin S, Brezault C, Delucinge-Vivier C, Descombes P, Dousset B, et al. Gene expression profiling provides insights into pathways of oxaliplatin-related sinusoidal obstruction syndrome in humans. Mol Cancer Ther. 2011;10: 687-96.

23. Agostini J, Benoist S, Seman M, Julie C, Imbeaud S, Letourneur $\mathrm{F}$, et al. Identification of molecular pathways involved in oxaliplatin-associated sinusoidal dilatation. J Hepatol. 2012; 56:869-76.

24. Tamandl D, Klinger M, Eipeldauer S, Herberger B, Kaczirek $\mathrm{K}$, Gruenberger B, et al. Sinusoidal obstruction syndrome impairs long-term outcome of colorectal liver metastases treated with resection after neoadjuvant chemotherapy. Ann Surg Oncol. 2011;18:421-30.

25. Vreuls CP, Van Den Broek MA, Winstanley A, Koek GH, Wisse E, Dejong $\mathrm{CH}$, et al. Hepatic sinusoidal obstruction syndrome (SOS) reduces the effect of oxaliplatin in colorectal liver metastases. Histopathology. 2012;61:314-8. 